\title{
Why does tuberculosis lead to specific inflammation?
}

\author{
Isamu SUGAWARA * \\ The Research Institute of Tuberculosis, Kiyose, Tokyo, Japan
}

[Received / Accepted: 2 June, 2009]

Key words : genome, immunity, M.tuberculosis, specific inflammation, virulence

When Mycobacterium tuberculosis infects humans, about $20 \%$ of those infected actually develop tuberculosis (TB) ${ }^{1)}$. In Japan, the incidence of TB in 2008 was 24,760 cases $(19.4 / 100,000$ persons) and the rate has been decreasing gradually, but is still higher than in the USA, Holland, and Belgium, for example. Histologically, tuberculosis displays exudative inflammation, proliferative inflammation and productive inflammation depending on the time course. In productive inflammation, granulomatous lesions with necrotic centers are formed. The typical granulomas consist of epithelioid macrophages, Langhans' multinucleated giant cells, lymphocytes and fibroblasts, and the process of their formation involves many cytokines, chemokines and transcription factors. These findings have been derived primarily from animal experiments utilizing an airborne infection apparatus. The conditions for airborne infection have been described in detail elsewhere ${ }^{2}$. This mini-review focuses on what has been found through animal experiments, and also indicates areas for which data are not currently available.

\section{Inflammatory process of tuberculosis}

Using animal experiments and an inhalation exposure system, the pathologic condition of the infected animals was followed up for one year. Exudative inflammation was observed for the first 10 days. Thereafter, granulomas, which corresponded to foci of proliferative inflammation,

\footnotetext{
*Corresponding author:

The Research Institute of Tuberculosis, 3-1-24 Matsuyama, Kiyose, Tokyo 204-0022, Japan. TEL : +81 424935075 FAX : +81-42-492-4600

E-mail : sugawara@jata.or.jp
}

were formed. Cavity formation was not recognized in animal tuberculosis, except for rabbits ${ }^{3)}$.

\section{Stages of experimental tuberculosis}

Dr. Arthur Dannenberg was one of the pioneers of experimental tuberculosis research.

Using rabbit models, he described the pathology of tuberculosis in detail ${ }^{3)}$. There are five stages: onset, symbiosis, early stages of caseous necrosis, interplay of cell-mediated immunity and tissuedamaging delayed-type hypersensitivity, and liquefaction and cavity formation. In stage 1, tubercle bacilli are usually destroyed or inhibited 
by the mature resident alveolar macrophages that ingest them. If bacilli are not destroyed, they grow and eventually destroy the alveolar macrophages. In stage 2, bacilli grow logarithmically within the immature nonactivated macrophages. These macrophages enter a tubercle from the bloodstream. This stage is termed symbiosis because bacilli multiply locally without apparent damage to the host, and macrophages accumulate and divide. In stage 3 , the stage at which caseous necrosis first occurs, the number of viable bacilli becomes stationary because their growth is inhibited by the immune response to tuberculin-like antigens released from bacilli. Stage 4 is the stage that usually determines whether the disease becomes clinically apparent. Cell-mediated immunity plays a major role in this situation. The cytotoxic delayedtype hypersensitivity immune response kills these macrophages, causing enlargement of the caseous center and progression of the disease. If good cellmediated immunity develops, a mantle of highly activated macrophages surrounds the caseous necrosis. In stage 5, bacilli evade host defenses. When liquefaction of the caseous center occurs, the bacilli multiply extracellularly, frequently attaining very large numbers. The high local concentration of tuberculin-like products derived from these bacilli causes a tissue-damaging delayed-type hypersensitivity response that erodes the bronchial wall, forming a cavity. Liquefaction and cavity formation do not occur at all in mice and rats.

\section{Immune system involvement in experimental tuberculosis}

$\mathrm{T}$ cells can be divided into two subsets, Th1 and Th2, on the basis of the cytokines they produce. In tuberculosis, Th1 plays a major role in defense against tuberculosis. Th1 cells suppress Th2 cells, and IFN- $\gamma$ down-regulates Th2 responses. We do not know the role played by B cells in tuberculosis. When activated, B cells increase the production of IFN- $\gamma$ by NK cells, and via antibody-dependent cell-mediated cytotoxicity (ADCC) they confer on NK cells the specific function of killing bacilliladen macrophages. The exact role of CD25 T cells and $y / \delta$ T cells remains unknown because the cell populations in the granulomatous lesions are very low. As long as NK T cell knockout mice are used, NK T cells do not play a major role in defense against $\mathrm{TB}^{4}$.

\section{Role of neutrophils in the initial phase of tuberculosis}

The role of neutrophils in the development of tuberculosis remained unknown for a long time. We utilized lipopolysaccharide (LPS)-induced transient neutrophilia in the lungs. LPS $(50 \mu \mathrm{g} / \mathrm{ml})$ was administered intratracheally to male Fischer rats ${ }^{5}$, which were then infected with $M$. tuberculosis via an airborne route. Intratracheal injection of LPS significantly blocked the development of pulmonary granulomas and significantly reduced the number of pulmonary colony-forming units (CFU). Treatment with amphotericin B (an LPS inhibitor) or neutralizing anti-rat neutrophil antibody reversed the development of pulmonary lesions. LPS-induced transient neutrophilia prevented early mycobacterial infection. The timing of LPS administration was important. When given intratracheally at least 10 days after aerial infection, LPS did not prevent the development of tuberculosis. Neutrophils obtained by bronchoalveolar lavage killed $M$. tuberculosis bacilli. These results indicate clearly that neutrophils participate actively in defense against early-phase tuberculosis. 


\section{Characteristics of Langhans' multinucleated giant cells}

When we infected IFN- $\gamma$ knockout mice with avirulent H37Ra or BCG Pasteur, we found multinucleated giant cells in the granulomatous lesions. The lesions also contained tubercle bacilli and consisted of multinucleated cell clusters, being immunopositive with anti-Mac-3 antibody ${ }^{6}$. We subsequently infected many knockout mice with M. tuberculosis, but no Langhans' multinucleated giant cells were recognized. Thus it appears that formation of multinucleated giant cells requires optimal combinations and concentrations of various cytokines, and the level of IFN- $\gamma$, at least, has to be significantly low.

\section{Significance of IFN- $\gamma$ and TNF- $a$ in protection against tuberculosis}

The technique of gene targeting (knockout) has swept through biomedical research. IFN- $\gamma$, TNF- $\alpha$, IRF-1, NF-IL6, NF- $\kappa$ B p50, STAT 1 and STAT 4 knockout mice succumbed to $M$. tuberculosis infection over time. There appears to be a cytokine and transcription factor hierarchy in experimental tuberculosis. The results indicate that these molecules play major roles in defense against the disease, IFN- $\gamma$ and TNF- $\alpha$ being the leading players in this respect ${ }^{7)}$. Fig. 1 shows the cytokine hierarchy in experimental tuberculosis.

\section{Nitric oxide (NO) and apoptosis in tuberculosis}

It is generally believed that nitric oxide (NO) plays a leading role in the killing of tubercle bacilli. In my experience, this is so in mouse and rat tuberculosis. However, it is ucertain whether NO produced by human mononuclear phagocytes can also kill tubercle bacilli. NO is regulated by inducible NO synthase (iNOS), and it has been reported that macrophages in the lungs of individuals with clinically active $M$. tuberculosis infection often express catalytically competent iNOS ${ }^{8}$. Of course, that does not exclude the possibility that other yet unknown molecules may be responsible for tubercle bacilli killing.

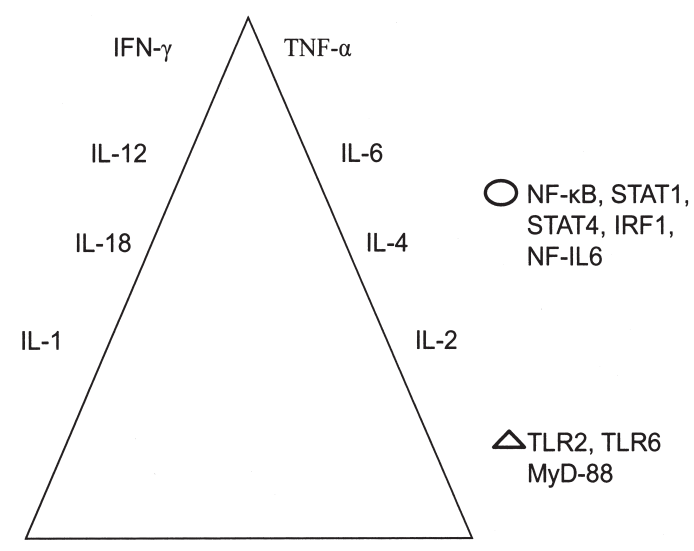

Fig. 1 Cytokine hierarchy in experimental tuberculosis.

IFN- $\gamma$ and TNF- $\alpha$ are "grand champions" (yokozuna in Japanese) in defense against tuberculosis. $\bigcirc$ : important molecules, $\triangle$ : less impotant molecules. 


\section{Alveolar macrophages in tuberculosis development}

When tubercle bacilli reach alveoli, they are phagocytosed by resident alveolar macrophages. Although tubercle bacilli are killed by alveolar macrophages, tubercle bacilli can also kill macrophages through an apoptotic process. What is the fate of tubercle bacilli once they enter the phagosomes of macrophages? We collected alveolar macrophages by bronchoalveolar lavage (BAL) from aerially infected guinea pigs. At 12 days after infection, one out of about 10,000 alveolar macrophages of various sizes contained many tubercle bacilli. This indicates that certain alveolar macrophages permit $M$. tuberculosis to replicate in the phagosomes, although most of the tubercle bacilli are killed by activated alveolar macrophages. It would be very interesting to examine the survival mechanism of $M$. tuberculosis at the single-cell level, but we still do not know why macrophages targeted by tubercle bacilli cannot kill bacilli. It is also known that macrophages possess an autophagy mechanism for removal of old organelles, in this case old infected phagosomes ${ }^{9)}$. Autophagic pathways can overcome the trafficking block imposed by M. tuberculosis .

\section{Exogenous reinfection and reactivation in tuberculosis}

Two different types of TB infection are recognized among elderly persons: exogenous reinfection, and reactivation of the same latent tubercle bacilli. As TB is still prevalent in Japan $(19.4 / 100,000$ persons), reactivation is common in elderly persons with the disease. However, it is not easy to demonstrate, because obviously it is not possible to obtain samples of $M$. tuberculosis isolated 20 or 30 years previously from the same patients. In America most researchers believe that exogenous reinfection is common because the prevalence rate of TB is so low.

Tubercle bacilli remain intact in macrophages for a long time without manifestation of TB. It is therefore important to understand the immunological aspects of TB pathogenesis under conditions of such dormancy. Latent tuberculosis is currently a topic of active discussion. There are few data on the mechanisms of latent tuberculosis in humans, but an understanding of them is very important in terms of pathogenesis and chemotherapy. As a first step, we used GFP-H37Rv M. tuberculosis to establish latent tuberculosis in guinea pigs, which are among the best animals for creating models of tuberculosis. We inoculated the guinea pigs subcutaneously with 100 or $1,000 \mathrm{CFU}$ of tubercle bacilli. During the 300-day follow-up period there were no clinical signs of disease. Upon necropsy no macroscopic lesions were recognized, but histopathological examination of major organs revealed microgranulomas consisting of epithelioid macrophages and lymphocytes without central necrosis. Interestingly, photon imaging visualized granulomatous lesions corresponding to these histologically apparent microgranulomas. Tuberculin skin testing of infected guinea pigs showed strong positivity ( $\geq 10 \mathrm{~mm}$ induration) throughout the entire experiment. Real-time PCR showed a slight increase in the expression of IFN- $\gamma$, TNF- $\alpha$, IL-12, and iNOS mRNAs. Our guinea pig model should be useful for examining the pathogenesis and molecular mechanisms of latent tuberculosis, as well as for monitoring the results of chemotherapy ${ }^{10)}$. 


\section{Present status of tuberculosis vaccine}

The efficacy of BCG against adult pulmonary tuberculosis still remains controversial. However, it is not so easy to find M. tuberculosis-derived immunogenic antigens suitable for TB vaccines because only a few such antigens have been detected so far. Several TB vaccines are currently being tested using various models ${ }^{11,12)}$. These include recombinant $\mathrm{BCG}$ vaccine expressing Ag85A, Ag85B, recombinant modified vaccinia virus Ankara expressing Ag85A, TB polyprotein vaccine, Mtb72f, ESAT-6 subunit vaccine, auxotrophic vaccines for $\mathrm{TB}$, and recombinant $\mathrm{BCG}$ overexpressing major extracellular proteins (rBCG30). Several promising TB vaccine candidates may become available after verification in monkey studies. We propose conducting a global multicenter study of the TB vaccine thus chosen using the same experimental protocols, and selecting a promising one on the basis of consensus.

\section{DNA genome of $M$. tuberculosis}

The genome of $M$. tuberculosis H37Rv has been sequenced ${ }^{13)}$, and the length of the DNA is reported to be $4.04 \mathrm{Mbp}$. It was hoped that this would allow complete clarification of the functions of $M$. tuberculosis, but this still remains a distant goal. Seven percent of the genome encodes proteins of unknown function and $26 \%$ encodes conserved hypothetical proteins. At present, there are not many proteins that are immunogenic and thus suitable for TB vaccine design. Another important point is that $M$. tuberculosis does not possess toxins. This is in sharp contrast to Listeria monocytogenes (a type of intracellular pathogen), which possesses listeriatoxin. Different protein antigens may be clarified in the near future.

\section{Cell wall components of} M. tuberculosis

The cell wall of M. tuberculosis consists of lipid and sugar moieties. As M. tuberculosis induces granulomatous lesions, we have tried to find granulomatogenic substances derived from tubercle bacilli. When four kinds of mycolates derived from M. tuberculosis Aoyama B strain were introduced into the lungs via an airborne route, only trehalose 6,6'-dimycolate (TDM) and methyl ketomycolate induced pulmonary granulomas without central necrosis. The pulmonary granulomas consisted of epithelioid macrophages and $\mathrm{T}$ lymphocytes. Although these substances hardly induce immune responses, they are granulomatogenic ${ }^{14)}$.

\section{Tuberculosis and high-risk factors}

There are many high-risk factors for TB, including HIV, malnutrition, aging and poverty. Diabetes mellitus (DM) is also one such factor. Although there is a clinical association between DM and tuberculosis, no experimental evidence for this association exists. We attempted to clarify whether type 1 diabetic (KDP) and Goto Kakizaki type 2 diabetic rats were more susceptible to $M$. tuberculosis than non-diabetic wild-type (WT) rats. The infected diabetic rats developed large granulomas without central necrosis in their lungs, liver or spleen. This was consistent with a significant increase in the number of CFU of M. tuberculosis in the lungs and spleen $(p<0.01)$. Insulin treatment resulted in significant reduction of tubercle bacilli in the infected KDP rats $(p<0.01)$. Pulmonary IFN- $\gamma$, TNF- $\alpha$ and IL- $1 \beta$ mRNA levels were higher in the infected diabetic rats than 
in WT rats. Alveolar macrophages from KDP rats were not fully activated by $M$. tuberculosis infection because they did not secrete nitric oxide (NO) that can kill $M$. tuberculosis $(p<0.01)$. However, there was no significant difference in phagocytosis of tubercle bacilli by alveolar macrophages between KDP and WT rats. Taken together, it is appears that type 1 diabetic (KDP) and GK rats are more susceptible to $M$. tuberculosis that WT rats ${ }^{15,16)}$. Hypercholesterolemia and hyperlipidemia were not observed in these rats. We are now investigating the relationship between DM and TB exacerbation at TB specialist hospitals.

\section{Concluding remarks}

It now seems appropriate to point out several reasons why tuberculosis belongs to the category of specific inflammation. First, as mentioned above, M. tuberculosis does not possess toxin, while another typical intracellular pathogen, Listeria monocytogenes, does. Most would consider that toxins are responsible for acute inflammation. Second, the cell wall of $M$. tuberculosis consists of various sugars, polysaccharides and lipids. Generally, immune responses induced by these moieties are low, but some mycolic acid derivatives (trehalose dimycolate and methyl ketomycolate) are granulomatogenic factors. Lastly, the doubling time of M. tuberculosis is around 20-24 h, and it replicates very slowly, surviving in phagosomes of specific alveolar macrophages for a long time. We are currently planning a project to examine why $M$. tuberculosis replicates in certain alveolar macrophages at the single-cell level, a phenomenon that we have termed the death escape mechanism. It would also be interesting to examine the process (termed autophagy) by which infected macrophages remove old infected phagosomes.

Mycobacterial research is one of a number of fascinating areas that challenge us as professional researchers. I hope that the next generation of researchers will tackle many of the issues that still remain unresolved.

\section{References}

1) Enarson DA, Wang JS, Dirks JM: The incidence of active tuberculosis in a large urban area. Am J Epidemiol 129: 1268-1276, 1989.

2) Sugawara I, Yamada H, Otomo K, Aoki T, Mizuno S, Udagwa T: Optimal conditions for establishment of an experimental tuberculosis model using an automated inhalation exposure apparatus and its application. Kekkaku 7: 463469, 2000 .

3) Dannenberg AM: Pathogenesis of human pulmonary tuberculosis. Insights from the rabbit model. ASM Press, Washington, DC. 2006.

4) Sugawara I, Yamada H, Mizuno S, Li C, Nakayama T, Taniguchi M: Mycobacterial infection in natural killer $\mathrm{T}$ cell knockout mice. Tuberculosis 82: 97-104, 2002.

5) Sugawara I, Udagawa T, Yamada H: Rat neutrophils prevent the development of tuberculosis. Infect Immun 72: 1804-1806, 2004.

6) Sugawara I, Yamada H, Kazumi Y, Doi N, Otomo K, Aoki T, Mizuno S, Udagawa T, Tagawa $\mathrm{Y}$, Iwakura Y: Induction of granulomas in IFN-gamma gene-disrupted mice by avirulent but not by virulent strains of Mycobacterium tuberculosis. J Med Microbiol 47: 871-877, 1998.

7) Sugawara I, Yamada H, Shi R: Pulmonary tuberculosis in various gene knockout mice with special emphasis on roles of cytokines and transcription factors. Current Resp Rev 1: 7-13, 2005.

8) Nicholson S, Bonecini-Almeida Mda G, Lapa e Silva JR, Nathan C, Xie QW, Numford R, 
Weidner JR, Calaycay J, Geng J, Boechat N, Linhares C, Rom W, Ho JL: Inducible nitric oxide synthase in pulmonary alveolar macrophages from patients with tuberculosis. J Exp Med 183: 2293-2302, 1996.

9) Gutierrez MG, Master SS, Singh SB, Taylor GA, Colombo MI, Deretic V: Autophagy is a defense mechanism inhibiting BCG and $M y$ cobacterium tuberculosis survival in infected macrophages. Cell 119: 753-766, 2004.

10) Sugawara I, Mizuno S, Tatsumi T, Taniyama $\mathrm{T}$ : Imaging of pulmonary granulomas using a photon imager. Jpn J Infect Dis 59: 332-333, 2006.

11) Sugawara I, Li Z, Sun L, Udagawa T, Taniyama T: Recombinant BCG Tokyo (Ag85A) protects cynomolgus monkeys (Macaca fascicularis) infected with H37Rv Mycobacterium tuberculosis. Tuberculosis 87: 518-525, 2007.

12) Sugawara I, Sun L, Mizuno S, Taniyama T: Protective efficacy of recombinant BCG Tokyo (Ag85A) in rhesus monkeys (Macaca mulatta) infected intratracheally with H37Rv Mycobacterium tuberculosis. Tuberculosis 89: 62-67, 2009.

13) Cole ST, Brosch R, Parkhill J, Garnier T, Churcher C, Harris D, Gordon SV, Eiglmeier
K, Gas S, Barry, III CE, Tekaia F, Badcock K, Basham D, Brown D, Chillingworth T, Connor R, Davies R, Devlin K, Feltwell T, Gentles S, Hamlin N, Holroyd S, Hornsby T, Jagels K, Krogh A, McLean J, Moule S, Murphy L, Oliver K, Osborne J, Quail MA, Rajandream MA, Rogers J, Rutter S, Seager K, Skelton J, Squares R, Squares S, Sulston JE, Taylor K, Whitehead S, Barrell BG: Deciphering the biology of Mycobacterium tuberculosis from the complete genome sequence. Nature 393: 537544, 1998.

14) Sugawara I, Udagawa T, Hua S, Reza-gholizadeh M, Otomo K, Saito Y, Yamada H: Pulmonary granulomas of guinea pigs induced by inhalation exposure of heat-treated BCG Pasteur, purified trehalose dimycolate and methyl ketomycolate. J Med Microbiol 51: 131-137, 2002.

15) Sugawara I, Yamada H, Mizuno S: Pulmonary tuberculosis in spontaneously diabetic Goto Kakizaki rats. Tohoku J Exp Med 204: 135145, 2004.

16) Sugawara I, Mizuno S: Higher susceptibility of type 1 diabetic rats to Mycobacterium tuberculosis infection. Tohoku J Exp Med 216: 363370, 2008. 